\title{
Contraceptive practices of hiv-positive women who used prevention of vertical transmission of hiv services in south Africa
}

\begin{abstract}
Background: South Africa's reported prevalence of HIV among women using prenatal services ranged from $17 \%$ to 37.4 in various provinces during 2011, emphasizing the importance of these women's contraceptive practices. This study attempted to identify these practices so that future health education could address identified challenges.

Methods: Trained research assistants administered self-designed questionnaires to a conveniently selected sample of women who had used prevention of vertical transmission of HIV services, and attended well-baby clinics in the study area during the data collection phase.

Results: A minority of women used condoms consistently at every sexual encounter, exposing themselves to risks of HIV re-infections and to other infections, and increasing the risk of vertical transmission of HIV for their babies.

Conclusion: As more than $50 \%$ of the respondents did not desire to have more children, their contraceptive practices should be adjusted (by using dual contraception) to enable them to achieve this goal. Misconceptions about HIV and condoms, such as 'giving back HIV' to a sex partner by not using condoms, should also be addressed as a matter of urgency. Effective contraceptive practices could enhance the wellbeing of the HIV-positive women as well as their current and future babies. The outcomes of services striving to prevent vertical transmission of HIV, might be jeopardised by HIV-positive women's ineffective contraceptive practices.
\end{abstract}

Keywords: AIDS, autoimmune deficiency syndrome; condom use; contraceptives; human immune deficiency virus; HIV, prevention of vertical transmission of HIV
Volume 5 Issue I - 2017

\author{
VJ Ehlers, AN Mbokane, JH Roos \\ Department of Health Studies, University of South Africa, South \\ Africa
}

Correspondence: VJ Ehlers, Department of Health Studies, University of South Africa, PO Box 392, Muckleneuk Rand 0003, South Africa, Tel +27 I2 $3478287,+2784587$ 3303, Fax +27 I2 429 6688,Email ehlersjh@mweb.co.za

Received: December 29, 2016 | Published: January 30, 2017
Abbreviations: AIDS, auto-immune deficiency syndrome; ART, anti-retroviral treatment; ARVs, anti-retrovirals; HIV, human immune deficiency virus; $\mathrm{NDoH}$, national department of health of south africa; PCR, polymerase chain reaction; SSA, sub sahara africa; STI, sexually transmitted infection; VL, viral load; VCT, voluntary counselling and testing

\section{Introduction}

During 2012, an estimated $70 \%$ of the 34.3 million people ${ }^{1}$ living with Human Immuno-Deficiency Virus (HIV) globally, lived in Sub Sahara Africa (SSA). During the same year, South Africa' estimated HIV-positive population comprised 5.6 million persons. ${ }^{2-3}$ The HIVpositive prevalence among women attending prenatal clinics in South Africa, ranged from $17 \%$ to $37.4 \%$ in the country's nine provinces. ${ }^{4}$ Vertical (or mother-to-child) transmission of HIV can occur during pregnancy, the birth process and/or while breastfeeding. Free services to prevent vertical transmission of HIV were instituted in South Africa ${ }^{5}$ by the National Department of Health (NDoH) during 2001, commencing with free voluntary counselling and testing (VCT) services. Prevention of vertical transmission of HIV services include counselling about the effective use of condoms, and the provision of free condoms, to prevent HIV re-infections. Such re-infections could increase the woman's viral load (VL) and decrease her CD4 count, increasing the risk of vertical transmission of HIV. Free anti-retrovirals (ARVs) are provided to the mother and baby. Effective prenatal care, timeous referrals to specialist care if required, appropriate infant feeding options, and consistent use of condoms should ensure optimal health of mother and baby.
As free ARVs enable HIV-positive persons to live longer and healthier lives, HIV-positive women could have several pregnancies after being diagnosed HIV-positive. These women should be enabled to make informed contraceptive choices as these choices affect their own, as well as their current and potential future children's wellbeing. However, limited information could be traced about HIV-positive women's utilisation of free contraceptives in South Africa. Consequently the current study endeavoured to identify the contraceptive practices of HIV-positive women who had utilised prevention of vertical transmission of HIV services in one province in South Africa. Such knowledge could assist health care providers to provide relevant contraceptive-related information to women utilizing these services. Effective utilization of free contraceptives could help to enhance these women's quality of life by enabling them to decide if and when they desired another pregnancy. Consistent uses of condoms prevent HIV re-infections which could cause rapid deterioration of the woman's physical condition by increasing her VL and decreasing her CD4 count.

\section{Materials and methods}

A quantitative descriptive design was adopted to identify the contraceptive practices of women who had utilised prevention of vertical transmission of HIV services in one district in the Mpumalanga Province of South Africa. The target population comprised all women who used these services during 2010 and 2011, attended one of the participating well-baby clinics during the data collection phase, were willing to sign consent to participate in the study and for information to be used from their own as well as from their babies' medical records. All women who met these criteria were informed about and requested 
to consider participating in the study. Most eligible women refused to participate in the study, probably because they did not want to be identified as being HIV-positive. Data analysis commenced when 200 women's questionnaires had been completed by the end of September $2011(\mathrm{~N}=200)$.

The self-designed questionnaire was based on the guidelines and policies of the $\mathrm{NDoH}$ pertaining to the prevention of vertical transmission of HIV. The English questionnaire was translated into IsiZulu and the translation was approved by bilingual expert. Two experienced researchers, the statistician and five experts in the field of prevention of vertical transmission of HIV, agreed that every item in the questionnaire was relevant to aim of the study. These experts also graded every item on a 5-point Likert scale, with 5 indicating perfect relevance of the item to the research purpose and 1 indicating no relevance. Only items that scored an average of at least 4 (content validity index of .8) were retained in the questionnaire. This article focuses on information obtained in response to questions asked about the women's personal (biographic) characteristics as well as about their sexual and contraceptive practices during pregnancy and breastfeeding. The babies' HIV status and the women's CD4 counts were recorded from the medical records.

Permission to conduct the study was granted by the University of South Africa, the Mpumalanga Provincial Department of Health and the managers of the participating health facilities. Every potential respondent was informed about the nature and purpose of the study and could decide independently whether or not to participate in the study, without risking any negative consequences. Respondents were assured about the anonymity and confidentiality of their information. Ten trained research assistants, fluent in English and IsiZulu (the dominant local language), who had signed confidentiality agreements with the 2 nd author, completed the questionnaires by asking the same questions in the same sequence from the participating women during one-on-one sessions in private rooms. This was done because many women in the research area might have been unable to complete the questionnaires on their own. The questionnaire was pretested on 10 women who were excluded from participation in the actual study. They understood all the questions but recommended that more familiar words should be used in some phrases. This was implemented.

The Statistical Package for the Social Sciences (SPSS) version 20 and MSExcel 2010 were used to analyse the data. A statistician assisted with the data analysis and interpretation.

\section{Results and discussion}

\section{Biographic information}

Most respondents' ages ranged from 20 to 29, followed by those aged 30-39. Avert ${ }^{6}$ also reported that the HIV prevalence is high among South African women aged 20-34. These HIV-positive women's relative young ages indicate the dire necessity for using effective contraception to make informed decisions about future pregnancies.

In this study $55.0 \% \%(\mathrm{n}=110)$ of the respondents passed secondary school grades $8-11$, and $30.5 \%(\mathrm{n}=61)$ passed grade 12 , implying that $85.5 \%(\mathrm{n}=171)$ of the respondents had some secondary school education.

Consequently $14.5 \%(n=29)$ respondents did not progress beyond primary school level (seven years of schooling in South Africa) and might have been unable to understand the rationale underlying the expected interventions of services for preventing vertical transmission of HIV. Venkatesh et al., ${ }^{7}$ stated that a woman with a higher level of education, is more likely to get tested for HIV, than women with less education because understanding the principles of HIV requires insight based on relatively advanced cognitive capacities.

Most respondents (78.0\%; $\mathrm{n}=156)$ were unemployed. These women had to rely on someone (presumably their husbands or partners) for financial survival and for feeding their babies. Out of 44 respondents who were working 18 (40.9\%) earned between R500 and R900; 9 (20.5\%), earned between R1000 and R1999; 7 (15.9\%) earned between R2000 and R2999; 6 (13.6\%) earned above R3000, and $4(9.1 \%)$ earned less than R500 (1USD was equivalent to about R15 during December 2016). According to Avert, ${ }^{8}$ in SSA women of poor socio-economic status, who are supported by their partners, tend to be afraid of getting tested for HIV, due to anticipated repercussions. This was not the case in this study as the respondents were not financially independent but were tested for HIV.

Of the respondents, $36.0 \%(n=72)$ had one child, $33.5 \%(n=67)$ had two children, $16.5 \%(n=33)$ had three children while $9.5 \%(n=19)$ had four and $4.5 \%(\mathrm{n}=9)$ had five children. The number of children that each respondent had, and her financial status, might have influenced her wellbeing during pregnancy as she needed money for her family's and her own nutrition as well as for visiting the ANC clinic. As 30.5\% $(n=61)$ of the respondents had at least three children, and $78.8 \%$ $(n=156)$ were unemployed they might have encountered difficulties to meet their nutritional needs during pregnancy and breastfeeding, especially if they had to feed three or more children on their meagre incomes, which could have negatively affected their wellbeing. These findings emphasise the fact that these women needed knowledge to utilize free contraceptives to plan their future pregnancies.

Only $18.0 \%(n=36)$ of the respondents presented with varying degrees of normal CD4 cell counts of $600 \mathrm{~mm} 3$ and above, $9.5 \%$ $(\mathrm{n}=19)$ had CD4 cell counts between $600 \mathrm{~mm} / 3$ and $699 \mathrm{~mm} / 3$ while $8.5 \%(\mathrm{n}=17)$ had CD4 cell counts of $700 \mathrm{~mm} 3$ and higher. HIV-positive women with low CD4 counts should postpone future pregnancies until their CD4 counts have increased to sufficiently high levels (600 $\mathrm{mm} 3$ and above). Pregnancy reduces a woman's immune responses to prevent rejection of the foetus and this is aggravated by a low CD4 count, increasing the woman's risk for opportunistic infections during and after pregnancy. Any HIV-positive woman should use contraception to delay a future pregnancy until her CD4 count has increased sufficiently, her VL is undetectable and she should use a condom at every sexual encounter to avoid the risk of HIV re-infection. As only $62.0 \%(\mathrm{n}=124)$ of the current study's 200 respondents' partners knew about the respondents' HIV-positive status, $38.0 \%(n=76)$ lacked this knowledge which might have affected their willingness to use condoms.

\section{Respondents' utilisation of condoms during and after their pregnancies}

During their pregnancies merely $46 \%(n=92)$ of the respondents used condoms but only $50 \%(\mathrm{n}=46)$ used condoms consistently at every sexual encounter, $15.2 \%(\mathrm{n}=14)$ used condoms rarely, and $34.8 \%(n=32)$ used condoms sometimes, Respondents who did not use condoms at every sexual encounter, were exposed to HIV reinfections, and other sexually transmitted infections (STIs), with an increased risk of vertical transmission of HIV due to the reduced CD4 counts and increased VL resulting from infections acquired during sexual intercourse. ${ }^{8}$

Out of 162 respondents who responded to the question about their condom use after their babies' births, $48.1 \%(\mathrm{n}=78)$ used condoms at every sexual encounter, but $23.5 \%(n=38)$ sometimes used condoms, $14.8 \%(n=24)$ rarely used condoms and $13.6 \%(n=22)$ never did so. 
Out of the 200 respondents, $56.5 \%(\mathrm{n}=113)$ fully agreed that condoms were effective for preventing HIV, $28.0 \%(n=56)$ agreed and $15.5 \%$ $(n=31)$ disagreed. Women who do not perceive condoms as being effective for preventing HIV are unlikely to use condoms effectively. As $38.7 \%(\mathrm{n}=58)$ of the respondents never used condoms during pregnancy and $13.6 \%(n=22)$ never did so after their babies' births, they increased their risks of contracting STIs and HIV re-infections at every sexual encounter, increasing the risk of vertical transmission of HIV. Cairns. ${ }^{9}$ stated that inconsistent condom use amounts to no condom use at all since STIs and/or HIV re-infections can occur during every sexual encounter without using a condom. As $38.0 \%$ $(n=76)$ of the respondents did not disclose their HIV-positive status to their partners, they might find it difficult to use condoms regularly. According to Avert (2011), some women in SSA might lack the power to negotiate condom use because they are either economically dependent on their male partners and/or they lack power in terms of their culture and norms.

Out of 92 respondents who used condoms, $79.3 \%(n=73)$ used male condoms, whilst $20.7 \%(n=19)$ used female condoms. However, Better Health ${ }^{10}$ reported that female condoms were 'weird', bulky to carry around, unfeminine and noisy during sexual intercourse. Female condoms might be unaffordable. ${ }^{11}$ Odu \& Oluwasegun ${ }^{12}$ cited various factors that might discourage male condom use, including interference with the authenticity of sex, challenging partners' mutual trust and the association of condoms with persons' HIV-positive status.

Although $7.0 \%(n=14)$ of the current study's 200 respondents had rarely used condoms during pregnancy, $12.0 \%(\mathrm{n}=24)$ rarely did so after their babies' births. Condoms were used sometimes during pregnancy by $16.0 \%(\mathrm{n}=32)$ of the respondents and by $19.0 \%(\mathrm{n}=38)$ after their babies' births. Respondents who used condoms at every sexual encounter during pregnancy were $23.5 \%(n=47)$ during pregnancy and $39.0 \%(\mathrm{n}=78)$ after their babies' births. Thus regular condom use seemed to increase after the respondents' babies had been born (Figure 1).

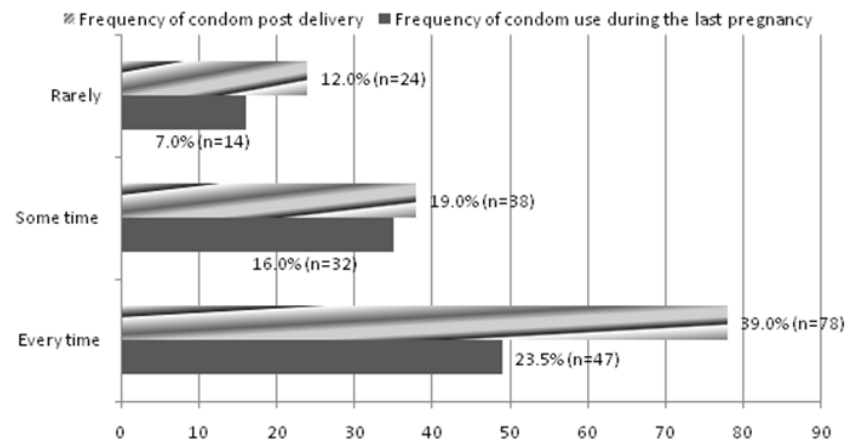

Figure I Comparison of condom usage during pregnancy and after the birth of the respondents' babies.

The risk of vertical transmission of HIV after the baby's birth exists if an HIV-positive woman breastfeeds, and therefore regular condom use remains an integral part of programs to prevent vertical transmission of HIV. Out of 150 respondents who had sexual relationships during pregnancy, only $31.3 \%(n=47)$ had used condoms consistently, implying that the babies of $68.7 \%(n=103)$ of these women were exposed to increased risks of vertical transmission of HIV during pregnancy, despite using services to prevent vertical transmission of HIV.

When respondents were asked if using a condom at every sexual encounter was difficult, $21.5 \%(n=43)$ agreed, while $10.5 \%(n=21)$ had reportedly never used condoms in their entire lives.
Based on this information, it cannot be explained, if using condoms at every sexual encounter was not a difficult process, why only $23.5 \%(\mathrm{n}=47)$ of the respondents used condoms consistently during pregnancy and only $39.0 \%(n=78)$ did so after the birth of their babies (Figure 1).

Out of the 200 respondents, $84.5 \% \quad(n=169)$ agreed that condoms prevent HIV infection during sexual intercourse as $42.6 \%$ $(\mathrm{n}=72)$ reported that condoms prevent HIV, STIs and pregnancy concurrently; $33.7 \%(\mathrm{n}=57)$ indicated that condom use prevents the spread of HIV and $23.7 \%(n=40)$ said that condoms prevent physical contact during sexual intercourse.

Out of 200 respondents, $68.0 \%(n=136)$ stated that condom use at every sexual encounter was not difficult as 136 respondents maintained that sex was the same with or without a condom $(42.6 \%$; $\mathrm{n}=58)$, one gets used to a condom $(21.3 \% ; \mathrm{n}=29)$, their partners did not have problems with condom use $(9.2 \% ; \mathrm{n}=26)$, and sex was still enjoyable even if a condom was used $(16.9 \% ; n=23)$.

According to 43 women who responded to this item, barriers to condom use included partners who do not want to use condoms (29.3\%; $\mathrm{n}=17)$, being afraid to disclose their HIV-positive status to their partners $(25.9 \% ; n=15)$, not wanting their partners to find out about their HIV-positive status $(17.2 \%$; $n=10)$; never discussing condom use $(12 / 1 \% ; n=7)$ and believing that the women were transferring HIV back to their male partners by not using condoms $(15.5 \%$; $n=9)$. Akelo et al. ${ }^{13}$ emphasised that the strategies for improving condom use, target women assuming that women have the power to implement condom use. The current study respondents $(51.2 \% ; n=22)$ confirmed that men controlled condom use. Thus teaching HIV-positive pregnant women about condom use might produce limited results without the cooperation of these women's partners. Misconceptions about the transmission of HIV also need to be addressed during counselling sessions. A few women apparently thought once a person suffering from HIV had infected another person, and then he/she would be cured of HIV. It remains uncertain precisely what the respondents implied when they indicated that they did not use condoms so that they could give HIV back to their male partners who had infected them. Future research should investigate this issue. Nevertheless health counsellors should emphasise that nobody gets cured from HIV by infecting other people and those HIV re-infections could have serious consequences for women and their babies.

\section{Respondents' utilisation of contraceptives}

Out of 200 respondents $66.0 \%(n=132)$ used contraceptive injections, 9.5\% $(\mathrm{n}=19)$ used contraceptive pills, $13 \%(\mathrm{n}=26)$ used barrier methods, while $5.5 \%(\mathrm{n}=11)$ of the women had been sterilised and $6.0 \%(\mathrm{n}=12)$ used no contraceptive.

The current study's 200 respondents indicated that the following contraceptive methods are suitable for HIV-positive women: $52.5 \%$ $(\mathrm{n}=105)$ injections, $8 \%(\mathrm{n}=16)$ pills. $24.0 \%(\mathrm{n}=48)$ condoms, $7.5 \%$ $(\mathrm{n}=15)$ dual method, $2.5 \%(\mathrm{n}=5)$ sterilization and $5.5 \%(\mathrm{n}=11)$ did not know. Cothran, ${ }^{14}$ as well as Blish \& Baeten, ${ }^{15}$ stipulated that, if HIVpositive women are to be safe from HIV, STIs and pregnancy, dual protection should be utilised. Dual protection refers to using a condom with either contraceptive pills or injections. This ensures protection against pregnancy in cases where condoms break. The majority of the current study's HIV-positive respondents $(92.5 \%$; $n=185)$ might not have mentioned dual protection as a contraceptive method of choice due to a lack of information and/or inadequate counseling. ${ }^{16}$ 


\section{Respondents' perceptions about dual contraception}

When the 200 respondents were asked if dual protection was important, 97\% $(n=194)$ agreed, only 3.0\% $(n=6)$ disagreed. Out of the 194 respondents who indicated that dual protection was important, $50.5 \%(\mathrm{n}=98)$ stated that one would be protected from pregnancy in case a condom broke, $44.8 \%(n=87)$ indicated that a woman would be protected from pregnancy even if the male partner refused to use a condom, $4.6 \%(n=9)$ mentioned that condoms have a tendency to burst. No respondent mentioned protection from HIV re-infection and from STIs in response to this question.

Those respondents $(3.0 \% ; n=6)$ out of the total sample of 200 women, who did not perceive dual protection to be important, mentioned that total safety from pregnancy was possible only through abstinence. However, none of these six women managed to practise abstinence because they had all given birth to their babies prior to being interviewed by the current study's research assistants.

Some contradictions became apparent when the respondents' replies to specific questions were compared. Only $13.0 \%(n=26)$ of the respondents said they were using barrier contraceptive methods after the birth of their babies, and only $7.5 \%(n=15)$ mentioned the dual method as a suitable contraceptive method for HIV-positive women. However, $97.0 \%(\mathrm{n}=194)$ of the 200 respondents agreed that the dual method is the ideal contraceptive method for HIV-positive women. Thus respondents in the current study appeared to know about the importance of using dual protection, but they chose not to use it. Nakaie et al., ${ }^{17}$ cited lack of knowledge as one of the reasons why HIV-positive women ended up being pregnant, but this was not the case for $97 \%(n=94)$ of the respondents in the current study.

In responses to the question about the number of children that each woman still wanted to have in future, the respondents replied as follows: $51.0 \%(n=102)$ did not want another child; $17.5 \%(n=35)$ wanted to have one more child; $15.0 \%(\mathrm{n}=30)$ wanted two more children; $5.0 \%(\mathrm{n}=10)$ wanted three more children and $2.5 \%(\mathrm{n}=5)$ wanted four more children while $3.5 \%(n=7)$ were unsure. Out of the 200 respondents, $5.5 \%(\mathrm{n}=11)$ had been sterilised.

Chances of HIV re-infection exist because condoms cannot be used while attempting to become pregnant. According to Aidsmap, ${ }^{18}$ the viral load of the couple, if they are both infected, or even if only one is infected, will determine the transmission risk, from one partner to the other, and from mother to child. The lower the VL, the lower the risk of vertical transmission of HIV during pregnancy and child birth. ${ }^{19}$

As $51.0 \%(\mathrm{n}=102)$ of the 200 respondents desired no more children, they might have been willing to consider sterilisation. In response to a specific question $60.5 \%(\mathrm{n}=121)$ respondents were indeed willing to be sterilised, $34.0 \%(\mathrm{n}=68)$ were not interested, whilst $5.5 \%(\mathrm{n}=11)$ had already been sterilised.

It could not be explained, based on the available information obtained during the 200 structured interviews, why $51.0 \%(n=102)$ of the respondents did not desire to have more children while $60.5 \%$ $(n=121)$ were willing to be sterilised. The item did not specify the anticipated time of sterilisation. Thus some of these women might have been willing to consider sterilisation only after they had born their desired number of children. However, this could not be supported nor refuted on the basis of the available information.

The 105 respondents, who preferred contraceptive injections, mentioned the following benefits: $41.9 \%(n=44)$ said it was easier to forget a contraceptive pill than an injection; $33.3 \%(n=35)$ perceived an injection as always being in the blood stream protecting the user against pregnancy; $7.6 \%(\mathrm{n}=8)$ believed injections to be more effective than contraceptive pills; $6.7 \%(n=7)$ believed if the respondents were on dual protection, when condoms broke, the injection would prevent pregnancy; $4.8 \%(n=5)$ stated that contraceptive pills could not be taken concurrently with ARVs, and $5.7 \%(n=6)$ stated that men did not want to use condoms.

Only $2.5 \%(\mathrm{n}=5)$ of the respondents perceived it to be best for an HIV-positive woman to be sterilised. They mentioned that it was not a good thing to have more children if you were HIV-positive. However, $121(60.5 \%)$ women said they were willing to be sterilised, but they were responding to a different question, which asked only whether they would be willing to be sterilised without specifying the time or conditions for sterilizations to be acceptable.

When the respondents were asked if they had experienced challenges when using contraceptives, $83.0 \%(\mathrm{n}=166)$ did not report any such challenges, and $11.0 \%(\mathrm{n}=22)$ did, whilst $6 \%(\mathrm{n}=12)$ stated that they were not using any contraceptives.

\section{Challenges experienced during contraceptive use}

Several challenges were reportedly experienced by $22(11.0 \%)$ respondents while using contraceptives, as shown in Table 1. A total of $94 \%(n=188)$ of the respondents utilised contraceptives to prevent pregnancies. Factors that could have modified the respondents' contraceptive use were the number of children that each respondent wanted to have, their perceptions of dual protection and their desire to be sterilised. Several benefits of the various contraceptive methods were identified.

Table I Challenges experienced by respondents when using specific contraceptive methods $(n=22)$

\begin{tabular}{lll}
\hline Types of Challenges Experienced during Contraceptive Use $\mathbf{n}$ & $\%$ \\
\hline Injections & & \\
\hline Headaches & 9 & 40.9 \\
Dizziness & 5 & 22.7 \\
Amenorrhea & I & 4.5 \\
\hline Pills & & \\
\hline Headaches & 2 & 9.1 \\
Nausea & 1 & 4.5 \\
\hline Male Condoms & & \\
\hline Breaking during Sexual Intercourse & 2 & 9.1 \\
\hline Female Condoms & & \\
\hline Noisy during Sexual Intercourse & 2 & 9.1 \\
Total & 22 & 99.9
\end{tabular}

\section{Conclusion}

Although $94.4 \%(\mathrm{n}=188)$ of the respondents utilised contraceptives only $13.0 \%(n=26)$ used barrier methods. Any HIV-positive woman should use dual contraception, including a condom to provide a barrier against HIV transmission and protection against pregnancy in case a condom breaks. Only 7.5\% $(\mathrm{n}=15)$ of the respondents mentioned that dual protection was a suitable contraceptive method for HIVpositive women. The importance of using dual contraception should be emphasized during future counselling sessions with HIV-positive pregnant women.

HIV-positive women require sufficient contraception knowledge to make informed decisions as $40.0 \%(\mathrm{n}=80)$ of the respondents desired to have more children ranging from 1 to $4 ; 51.0 \%$ did not want more children, $5.5 \%$ had been sterilised, and 3.5\% were unsure about whether they wanted more children Health counsellors should 
address challenges of using contraceptives as $11.0 \%(n=22)$ of the respondents reported contraceptive challenges including nausea, headaches, dizziness, and amenorrhoea, breakage of male condoms and noisiness of female condoms. Misconceptions about HIV and condoms use, such as 'giving back' HIV to one's male partner by not using a condom, should be addressed during counselling sessions.

Only a minority of the current study's respondent's used condoms consistently at every sexual encounter while pregnant and breastfeeding. Re-infection with HIV while pregnant or breast feeding, could increase the woman's viral load and decrease her CD4 count, increasing the risk of vertical transmission of HIV to her baby. Administering expensive ARVs to the mother and infant, and providing other free services to prevent vertical transmission of HIV, might be counterproductive unless these HIV-positive women use condoms consistently at every sexual encounter while pregnant and breastfeeding. Effective utilization of condoms at every sexual encounter could a cost-effective way of enhancing the outcomes of services to prevent vertical transmission of HIV. Thus health counselling could enhance HIV-positive women's effective contraceptive practices with definite benefits for the women themselves, as well as for their current and future babies.

\section{Acknowledgements}

The research, on which this article is based, was conducted for the 2nd author's doctoral thesis at the University of South Africa. The contributions of the research assistants are acknowledged for collecting the required information and the participating women for sharing their experiences. The relevant health care authorities are thanked for allowing data to be collected at the participating wellbaby clinics.

\section{Conflicts of interest}

None.

\section{Funding}

None.

\section{References}

1. World Health Organization. Consolidated guidelines on the use of antiretroviral drugs for treating and preventing HIV infection. 2013.

2. World Health Organization. HIV/AIDS Fact sheets. 2013.
3. Amfar. Statistics: Women and HIV/AIDS. 2013.

4. National Department of Health. The 2011 national antenatal sentinel HIV \& syphilis prevalence survey in South Africa. Pretoria: Government Printer. 2012.

5. National Department of Health. The South African antiretroviral treatment guidelines. Pretoria: Government Printer. 2013.

6. Avert. HIV and Aids in South Africa. 2014.

7. Avert. Preventing mother-to-child transmission (PMTCT) in practice. 2013.

8. Avert. Preventing mother-to-child transmission (PMTCT) of HIV. 2011.

9. Cairns G. Consistent condom use stops $70 \%$ of HIV infections, says CDC. 2013.

10. Better Health. Contraception-condoms for women. 2014.

11. Dawn SM. Everything you need to know about male condoms. 2014.

12. Odu BK, Oluwasegun GF. Condom use among a high risk behaviour group in Ekiti-State, Nigeria. International Journal of Tropical Medicine. 2011;6(1):8-14.

13. Akelo V, Girde S, Borkowf CB, et al. Attitudes toward family planning among HIV-positive pregnant women enrolled in a prevention of mother-to-child transmission study in Kisumu, Kenya. PLoS One. 2013;8(8):e66593.

14. Cothran D. Women shouldn't be sneaky to get family planning they want. 2011.

15. Blish CA, Baeten JM. Hormonal contraception and HIV-1 transmission. American Journal of Reproductive Immunology. 2011;65(3):302-307.

16. Clayden P. High rates of pregnancy in HIV-positive women in Zambia and South Africa. 2014.

17. Nakaie N, Tuon S, Ikuma N, et al. Family planning practice and predictors of risk of inconsistent condom use among HIV-positive women on anti-retroviral therapy in Cambodia. BMC Public Health. $2014 ; 14: 170$.

18. Aidsmap. The link between sexually transmitted infections and HIV. 2014.

19. Warszawski J, Tubiana R, Le Chenadec J, et al. Mother-to-child HIV transmission despite antiretroviral therapy in the ANRS French perinatal cohort. AIDS. 2008;22(2):289-299. 